\title{
Efficacy and Safety Aiming at the Combined-Modality Therapy of External Beam Radiotherapy (40Gy) and lodine- I 25 Seed Implantation for Locally Advanced NSCLC in the Elderly
}

Li-Jun Tian, ${ }^{1, *}$ Hong-Zhi Liu, ${ }^{2, *}$ Qiang Zhang,' Dian-Zhong Geng,' Yu-Qing Huo,' Shou-Jian Xu,' Yan-Zhang Hao'

'Department of Oncology, Binzhou Medical University Hospital, Binzhou, Shandong, People's Republic of China; ${ }^{2}$ Department of Orthopedics, Binzhou Medical University Hospital, Binzhou, Shandong, People's Republic of China

*These authors contributed equally to this work

Video abstract

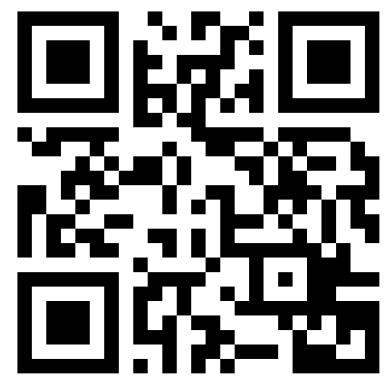

Point your SmartPhone at the code above. If you have a $Q R$ code reader the video abstract will appear. Or use: https://youtu.be/lumH-anM-SO

Correspondence: Yan-Zhang Hao HuangHe Two Road, Binzhou, 256603 Tel +8615506308710

Fax +86 (0)543-3257792

Email byfyhyz@bzmc.edu.cn
Purpose: To evaluate the efficacy and safety of combined-modality therapy for elderly patients with locally advanced non-small-cell lung cancer (NSCLC) invading the chest wall. Patients and Methods: We retrospectively enrolled 21 elderly patients (aged $\geq 60$ years) with locally advanced NSCLC invading the chest wall. For external beam radiotherapy (EBRT) of the primary tumor, 40Gy was applied and supplemented with iodine-125 seed implantation while $60 \mathrm{~Gy}$ was applied to the lymph nodes of the mediastinum. Follow-up was conducted every 3 months postoperatively. The related analytic parameters were change in tumor size, the objective response rate (ORR), the disease control rate (DCR), the degree of pain relief, the improvement of physical status, and toxicity.

Results: The combined-modality therapy significantly inhibited local growth of the tumor (from $7.84 \pm 1.20$ to $4.69 \pm 1.90 \mathrm{~cm})(P<0.0001)$, with $71.4 \%$ ORR and $90.5 \%$ DCR at 1 year. The cancer-related pain was significantly relieved $(P<0.05)$ and physical status was significantly improved $(P<0.05)$. No procedure-associated death or grade $>2$ irradiation-related adverse effects were reported in this study.

Conclusion: The combined-modality therapy of EBRT with 40Gy and permanent iodine125 seed implantation is an efficacious and safe treatment option for elderly patients with locally advanced NSCLC invading the chest wall.

Keywords: iodine-125 seed implantation, external beam radiotherapy, combined therapy, NSCLC, elderly

\section{Introduction}

At present, lung cancer poses a major burden to human health, and the prevalence has been increasing over the years. ${ }^{1,2}$ In 2018, there were an estimated 2,090,000 newly diagnosed lung cancer patients and about 1,760,000 deaths. ${ }^{1}$ Lung cancer has the highest mortality rate, with more than half of the cases reported in Asia. ${ }^{1}$ In addition, lung cancer is more common among the elderly and the incidence increases with age. ${ }^{1}$ Approximately $20 \%$ of non-small-cell lung cancer (NSCLC) patients are suitable for curative resection, while patients presenting with advanced NSCLC are not able to undergo the surgery. ${ }^{3}$ Therefore the standard treatment options for locally advanced NSCLC patients without positive EGFR mutation or ALK-positive status are external beam radiotherapy (EBRT) and/or chemotherapy based on related guidelines and criterions. ${ }^{4}$ Considering the treatment toxicity and 
reduced effectiveness of chemotherapy, patients, especially for the elderly may be reluctant to undergo chemotherapy. ${ }^{5}$ To prevent damage to the surrounding normal tissues, an insufficient irradiation dose might be delivered to tumor lesions by EBRT, and the lung volume receiving at least 20Gy (V20) should be $<30 \%{ }^{6}$

The WHO divides old age in chronological order as follows: middle-aged (45-59 years old), elderly (60-74 years old), and advanced age (75 years or older). ${ }^{7}$ Consequently, people above the age of 60 are classified as elderly, and characterized by poor physical health and may prefer treatment with no side effects or minimal side effects. For elderly patients with accompanying poor general condition, they are more vulnerable to the side effects caused by chemotherapy or EBRT, such as myelosuppression, gastrointestinal reaction, radiation pneumonitis, and radiation esophagitis. Iodine-125 seed implantation, is a form of brachytherapy, that has gained significant attention from all over the world. ${ }^{2,8-10}$ There is a growing concern on the quality of life (QOL) among the elderly with locally advanced NSCLC, especially with regard to the option to be adopted. ${ }^{11}$ The iodine -125 seed particle is characterized by its small size: $0.5 \mathrm{~mm}$ diameter and $3.0 \mathrm{~mm}$ long, and a half-life decay time of 59.43 days. It can continuously release low- energy radiation which may rapidly fall off within $1.0 \mathrm{~cm} .{ }^{12}$ Similar to EBRT, an iodine- 125 seed implantation is a form of radiotherapy. However, it differs from EBRT, in that it is a form of brachytherapy used to deliver a high radiation dose (100-140Gy) to tumor lesions without damaging adjacent normal tissues. ${ }^{2}$ In addition, the introduction of computed tomography (CT) scanner has greatly improved the accuracy of implantation. Iodine -125 seed implantation takes less than $1-2$ hours. ${ }^{13}$ Additionally, the incidence of severe irradiation-related adverse effects is significantly reduced and the few complications disappear soon with supportive care. ${ }^{8}$ Considering the highlighted advantages mentioned, patients including the elderly, have shown great interest and good compliance to Iodine -125 seed implantation. ${ }^{14-17}$

In this retrospective study, we aimed at clarifying the efficacy and safety of combined-modality therapy, EBRT (40Gy) and iodine -125 seed implantation, for elderly patients with locally advanced NSCLC invading the chest wall. We also investigated the degree of pain relief caused by tumors invading the chest wall and the improvement of QOL after the combined-modality therapy.

\section{Patients and Methods}

\section{Patients}

Patients with locally advanced NSCLC invading the chest wall who received EBRT (40Gy) supplemented with iodine -125 seed implantation from January 2016 to February 2019 at Binzhou Medical University Hospital were retrospectively enrolled in this study. The pathological diagnosis of all patients was confirmed by bronchoscopy or needle biopsy according to the third edition of the World Health Organization guidelines. All patients underwent enhancedCT scanner or positron emission tomography-CT (PET-CT), brain enhanced magnetic resonance imaging (MRI) and bone emission computed tomography (ECT). Three experienced physicians performed TNM staging, according to the seventh edition of the American Joint Committee on Cancer. ${ }^{18}$ The inclusion criteria were as follows: patients had total lung V20 > 30\% when the prescription dose was $60 \mathrm{~Gy}$ (Figure 1) and V20 $<30 \%$ when the prescription dose was 40Gy; patients were diagnosed as locally advanced NSCLC (stage-IIIA/IIIB); patients aged $\geq 60$ years with Eastern Cooperative Oncology Group (ECOG) performance scores $\leq 3 ;{ }^{19}$ patients who had not previously received radiotherapy; primary tumor lesions invading the chest wall and causing cancer-related pain; tumor measuring $\leq 7 \mathrm{~cm}$; the estimated lifespan of patients was longer than 3 months; patients with normal coagulation, hepatorenal function and sufficient hematopoietic function; and patients who were resistant to undergo surgery. The exclusion criteria were as follows: patients with severe cardiopulmonary diseases or mental disorders; the interval between EBRT and iodine -125 seed implantation was longer than one week; patients who declined combined-modality therapy; the tumor size could not be measured in the lung; patients who could not cooperate during the puncturing treatment. This study was approved by the Ethics Committee of Binzhou Medical University Hospital. All procedures involving human participants were performed in accordance with ethical standards of the board and the 1964 Helsinki declaration and its later amendments. The written informed consent was obtained from all patients in this study.

\section{External Beam Radiotherapy (EBRT)}

Using the treatment planning system (TPS), the planning target volume (PTV) of all the tumor lesions was designed to deliver a prescription dose of 60Gy to the primary tumor with chest wall invasion and the lymph nodes of mediastinum using the intensity-modulated radiation therapy (IMRT). If the 


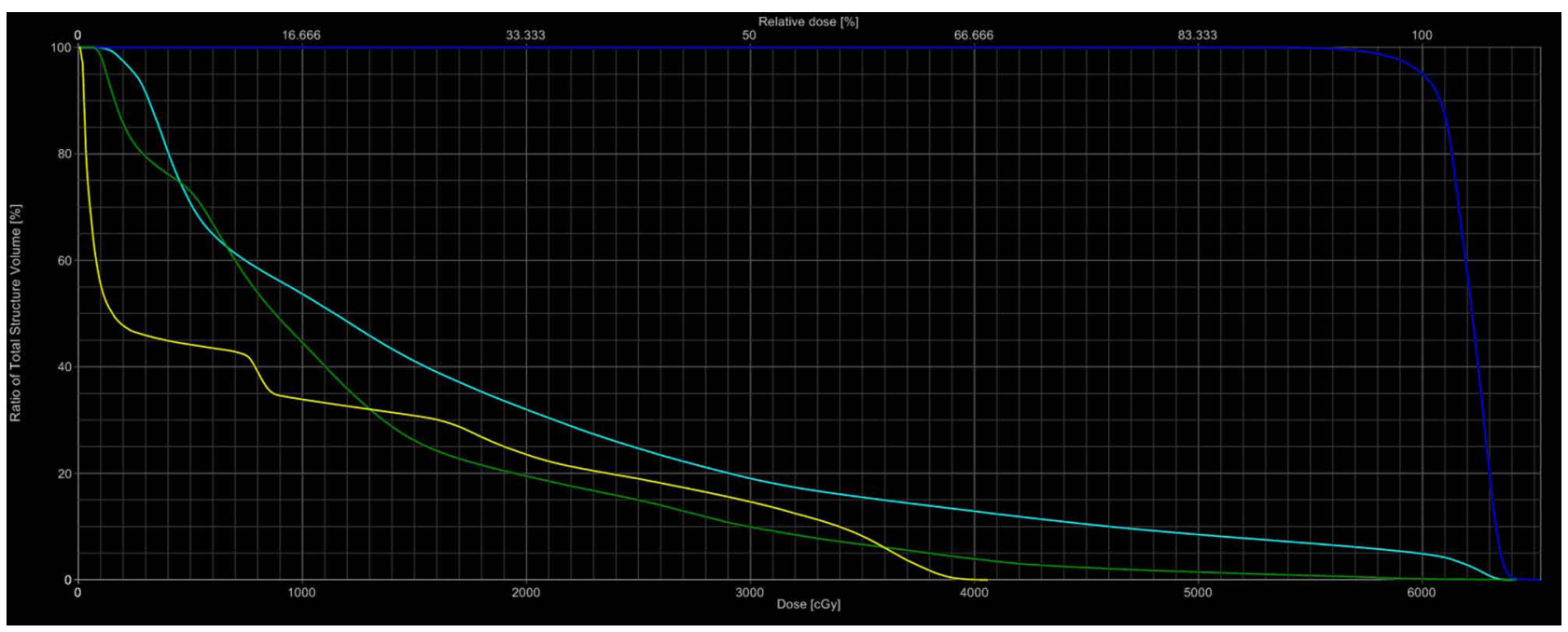

Figure I The Dose Volume Histogram of lung receiving a total radiotherapy dose of 60Gy. Blue line stands for the volume of lung receiving at different dose of radiotherapy; Yellow line stands for the volume of spinal cord at different dose of radiotherapy; Green line stands for the volume of heart at different dose of radiotherapy.

lung met the standard V20 $>30 \%$, the patient was enrolled in the study. When the EBRT of 40Gy was completed, iodine125 seed implantation for the primary tumor lesions was performed and EBRT for mediastinal lesions was continued according to the prior schedule. The prescription dose of $60 \mathrm{~Gy}$ was formulated to aim at the mediastinal lesions, while the primary tumor with chest wall invasion dose was a sum of 40Gy and the dose of iodine-125 seed implantation.

\section{lodine- 125 Seed Implantation}

\section{Eoperative Planning}

All patients underwent CT scan (Siemens Somatom Sensation 64 CT Scanner), after the EBRT prescription dose reached 40Gy. Subsequently, all obtained data was transmitted to a brachytherapy treatment planning system (BTPS; Linke, China) to complete the preoperative plan design. The procedure was as follows: delineating the GTV and adjacent organs at risk (OARs); formulating the PTV prescription dose; ascertaining the seed implantation pathway, such as the direction, depth and distribution; computing the quantity of iodine -125 seeds; and calculating the standardized dose constraints of OARs (eg lung $\mathrm{V} 20 \leq 30 \%$, heart V30<40\%). We defined $90 \%$ of the GTV dose (GTV D90) to meet the prescription dose. According to American Brachytherapy Society guidelines, the prescription dose for the combined-modality therapy was recommended to be $\geq 60 \mathrm{~Gy}^{20}$

\section{lodine- 125 Seed Implantation}

We adopted local infiltration anesthesia for all patients to relieve puncturing pain. Under the guidance of real-time
CT, experienced physicians, who would put on clothes made from lead ahead of time, performed the puncturing and seed implantation. The procedure was finished within 1-2 hours based on the preoperative designed plan, the predetermined pathway, and depth and distribution of each needle. After implantation, all patients repeatedly underwent CT scan to ascertain even distribution of iodine-125 seeds and whether there were any complications correlated to the implantation. After the operation, the patients were required to put on lead clothes for 6 months.

\section{Postoperative Dose Verification}

Postoperative CT scan images were transmitted to the BTPS to evaluate postoperative dosimetry. The physicist used D90 to calculate the tumor target dose. The OARs of thoracic tumors were defined as the lung, esophagus, heart, and spinal cord. The treatment procedure is shown in Figure 2.

\section{Efficacy and Safety Evaluation of Combined-Modality Therapy}

The corresponding index of local control rate, including the objective response rate (ORR), the disease control rate (DCR) and toxicity were evaluated in this study. The evaluation of efficacy was performed according to the Response Evaluation Criteria in Solid Tumors (RECIET) v $1.1^{21}$ as follows: CR (disappearance of the target lesion and lasting for at least 4 weeks), PR (more than 30\% decrease in the target lesion volume lasting for 4 weeks compared to the baseline), PD (at least 20\% increase of the target lesion volume from baseline lasting for 4 

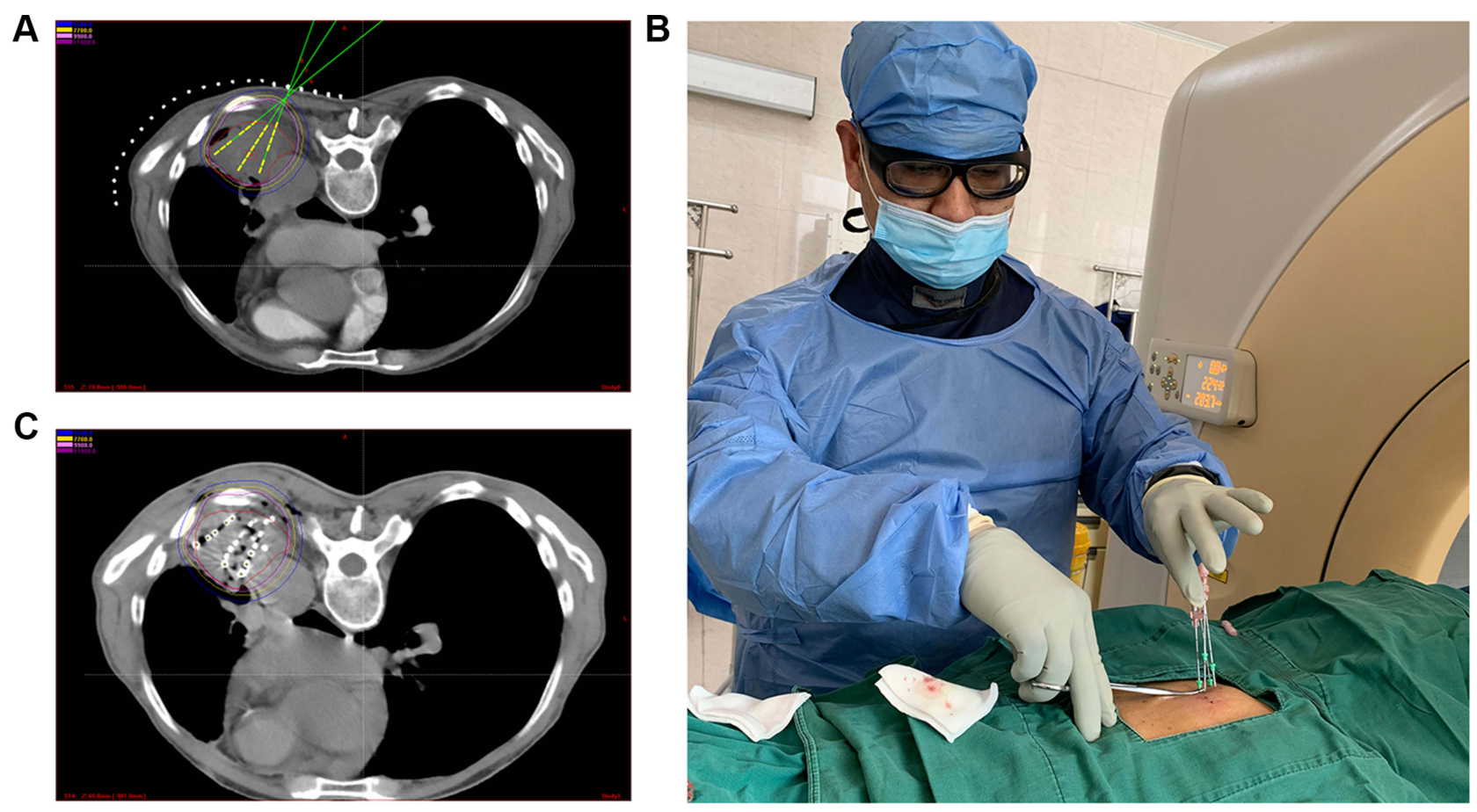

D

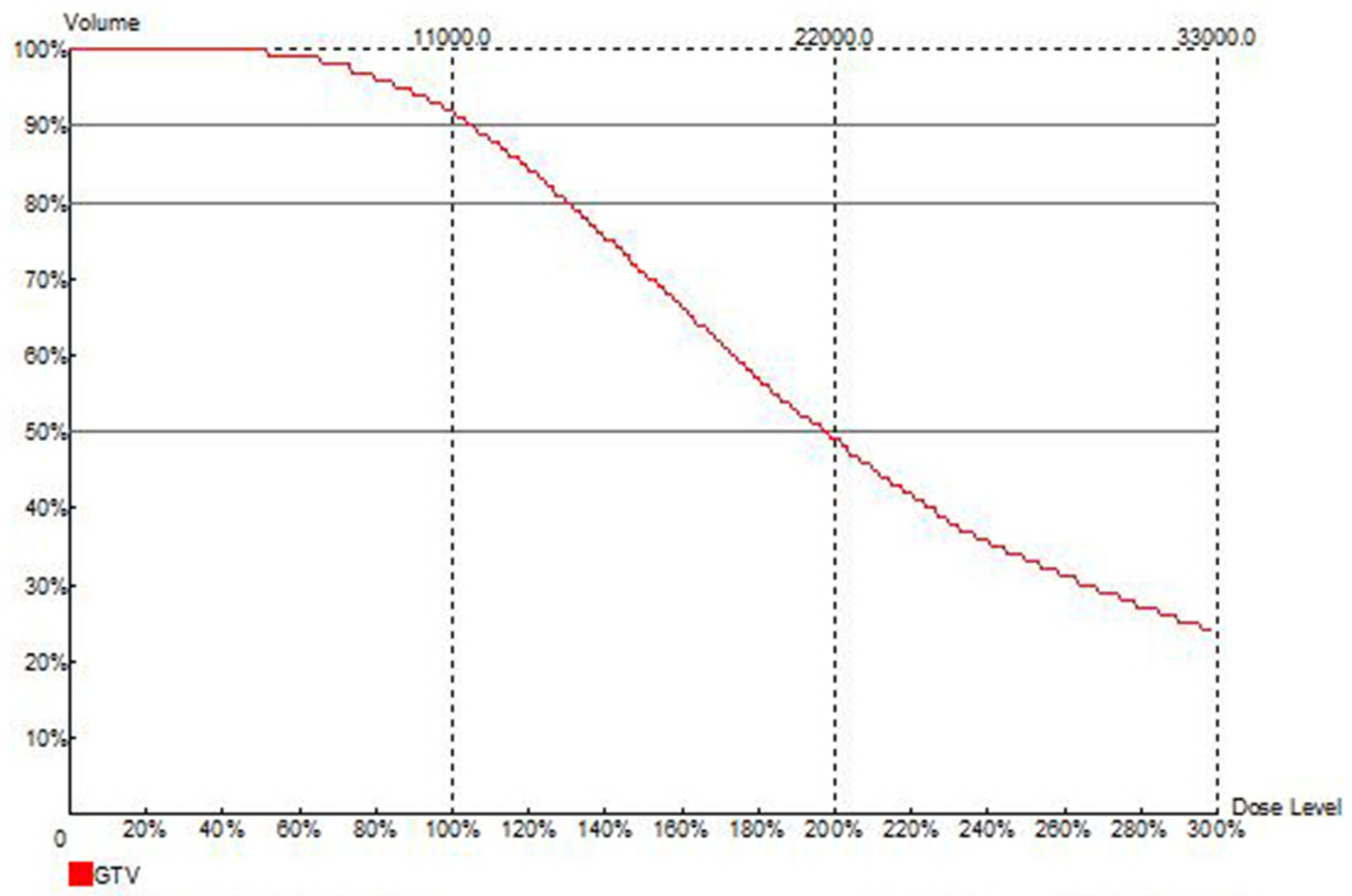

Figure 2 The treatment procedure. (A) Design of the preoperative plan, lines of different colors stand for different dose which was delivered to the primary tumor. (B) Course puncturing (C) The practical distribution of iodine -125 seed. (D) postoperative dose volume histography of tumor. 
weeks) and SD (between PR and PD). Objective response rate $(\mathrm{ORR})=(\mathrm{CR}+\mathrm{PR}) / \mathrm{all}$ cases $\times 100 \%$; and disease response rate $(\mathrm{DCR})=(\mathrm{CR}+\mathrm{PR}+\mathrm{SD}) /$ all cases $\times 100 \%$. The progression-free survival time (PFS) was defined as the time interval from the beginning of treatment to the occurring of disease progression, death or closure of the study. The overall survival (OS) time was calculated from the time of treatment to death. The irradiation-related acute and late adverse effects were evaluated according to the toxicity criteria of RTOG/EORTC (European Organization for Research and Treatment of CANCER). Based on the ECOG performance status, we classified performance status of the patients into five grades: 0 point indicated normal activity; 1 point indicated undertaking slight physical activity; 2 points indicated patients could move freely for more than half the day; 3 points indicated that patients lay in bed more than half the day; 4 points indicated that patients could not take care of themselves; 5 points indicated death. ${ }^{19}$ The numerical rating scale was used to calculate the pain score: 0 point indicated no pain, 1-3 points indicated slight pain, 4-6 points indicated moderate pain and 7-10 points indicated severe pain. $^{22}$

\section{Follow Up}

Patients were followed up for 3 months after the operation, and subsequently every 3 months for the first 2 years and every 6 months thereafter. During the follow-up period, if a patient had any uncomfortable symptoms, which were considered to be related to the tumor, a corresponding examination was performed. During the follow-up, CT scan, routine laboratory examination (eg blood cell count, tumor marker and hepatorenal function), evaluation of pain score, ECOG performance status and irradiationrelated adverse effects were performed.

\section{Statistical Analysis}

SPSS version 20 was used to analyze the data. Wilcoxon signed-rank test was used since the data did not comply with a normal distribution. Data were expressed as mean \pm $\mathrm{SD}$, the difference between before and after treatment was determined by the Student's $t$-test. Meanwhile, for categorical variables, the chi-square test was performed. $\mathrm{P}<0.05$ was considered statistically significant.

\section{Results}

A total of 21 patients who met the inclusion criteria were enrolled in this study. The characteristics and general information of all patients are listed in Table 1. As shown in Table 2, at 6-months follow-up, significant inhibition of local tumor growth was observed (the sum of the two: from $7.84 \pm 1.20$ to $4.69 \pm 1.90 \mathrm{~cm}) \quad(P<0.0001)$ (Figure 3C), after combined-modality therapy, obvious reduction in the maximum diameter of the tumor $(4.42$ \pm 1.28 vs $2.81 \pm 1.26 \mathrm{~cm})(P<0.001)$ (Figure $3 \mathrm{~A})$, and the maximum vertical diameter $(3.42 \pm 0.97$ vs $1.89 \pm 0.87 \mathrm{~cm})$ $(P<0.0001)$ (Figure 3B). The ORR and DCR were $76.2 \%$ and DCR $95.2 \%$ at 6-months, respectively. The ORR and DCR were 71.4 and $90.5 \%$ at 1 year, and 33.3 and $47.7 \%$ at 3 years, respectively. The OS rates were 62.3 and $23.9 \%$ at 1 year and 3 years, respectively. The cancer-related pain was observed in all the patients enrolled in our center before treatment (Table 3). Three patients achieved complete pain relief $(n=3), 12$ patients experienced moderate pain, two patients experienced partial pain relief, while four patients experienced severe pain. In this retrospective study, two of the three patients with $\mathrm{ECOG}=3$ moved freely more than half-day, and all patients with $\mathrm{ECOG}=2$ could undertake physical activity or move freely (Table 4). Cancer-related pain was significantly relieved $(P<0.05)$

Table I General Information of All Patients

\begin{tabular}{|l|l|l|}
\hline Characteristics & Patients & Percentage \\
\hline Sex & & \\
Male & 14 & 66.7 \\
Female & 7 & 33.3 \\
Age (years) & Median 68 (60-79) & \\
ECOG score & Median 2 (0-3) & \\
\hline Primary tumor stage & & \\
Stage IIIA & 3 & 14.3 \\
Stage IIIB & 18 & 85.7 \\
\hline Diameter of tumors (cm) & & \\
$<5.0$ & 4 & 19.0 \\
5.0-7.0 & 17 & 81.0 \\
Seed radioactivity & Median $0.64(0.47-0.82)$ & \\
Seed number & Median 76.4 (30-135) & \\
\hline Prescription dose & & \\
$<140$ Gy & 10 & 47.6 \\
$\geq 140 G y$ & 11 & 52.3 \\
\hline Short-term efficacy & & 5.5 \\
CR & 2 & 53.3 \\
PR & 14 & \\
SD & 5 & \\
\hline
\end{tabular}

Abbreviations: ECOG, Eastern Cooperative Oncology Group; Short-term efficacy, evaluate at 6 months after the implantation; CR, complete response; PR, partial response; SD, stable disease. 
Table 2 CT Scan Measurement Showing the Efficacy of Combined-Modality Therapy in Locally Advanced NSCLC Invading the Chest Wall Among the Elderly

\begin{tabular}{|l|c|c|c|}
\hline \multirow{2}{*}{ Groups } & \multicolumn{3}{|c|}{ Measurement of Tumors Through CT Scan (Mean \pm SD, cm) } \\
\cline { 2 - 4 } & Maximum Diameter & Maximum Vertical Diameter & cSum of the Two \\
\hline Before treatment & $4.42 \pm 1.28$ & $3.42 \pm 0.97$ & $7.84 \pm 1.20$ \\
After treatment & $2.81 \pm 1.26$ & $1.89 \pm 0.87$ & $4.69 \pm 1.90$ \\
Degree of free & 20 & 20 & 20 \\
T value & 4.118 & 5.340 & 5.230 \\
P-value & $P<0.001$ & $P<0.0001$ & $P<0.0001$ \\
\hline
\end{tabular}

Abbreviations: NSCLC, non-small-cell lung cancer; CT, computed tomography; SD, standard deviation; cm, centimeter; Sum of the two, the maximum diameter +the maximum vertical diameter.

and the cancer-related symptoms were also significantly improved $(P<0.05)$. The prescription dose in ten patients was delivered at $<140 \mathrm{~Gy}$, while in the remaining 11 patients it was delivered at $\geq 140 \mathrm{~Gy}$. The ORR at 1 year and 3 years for patients with a dose $\geq 140$ Gy were $81.8 \%$ and $45.4 \%$, respectively. The prescription dose $\geq 140 \mathrm{~Gy}$ was found to better inhibit local growth of tumor, but the difference was not significant because of the limited sample size. One patient was reported to have grade 1 radiation pneumonitis, with a slight dry cough which was relieved with supportive care. Two patients suffered from
Table 3 Analysis of Cancer-Pain Relief

\begin{tabular}{|l|l|l|l|l|l|}
\hline Group & \multicolumn{4}{|l|}{ Pain Score } & P-value \\
\hline & 0 & $1-3$ & $4-6$ & $7-10$ & \multirow{2}{*}{$P<0.05$} \\
\cline { 1 - 5 } Before treatment & 0 & 3 & 14 & 4 & \\
\cline { 1 - 4 } After treatment & 15 & 1 & 5 & 0 & \\
\hline
\end{tabular}

grade 1 radiation esophagitis which did not affect the food intake. Two patients suffered from slight pneumothorax and were gradually absorbed without any symptoms. No
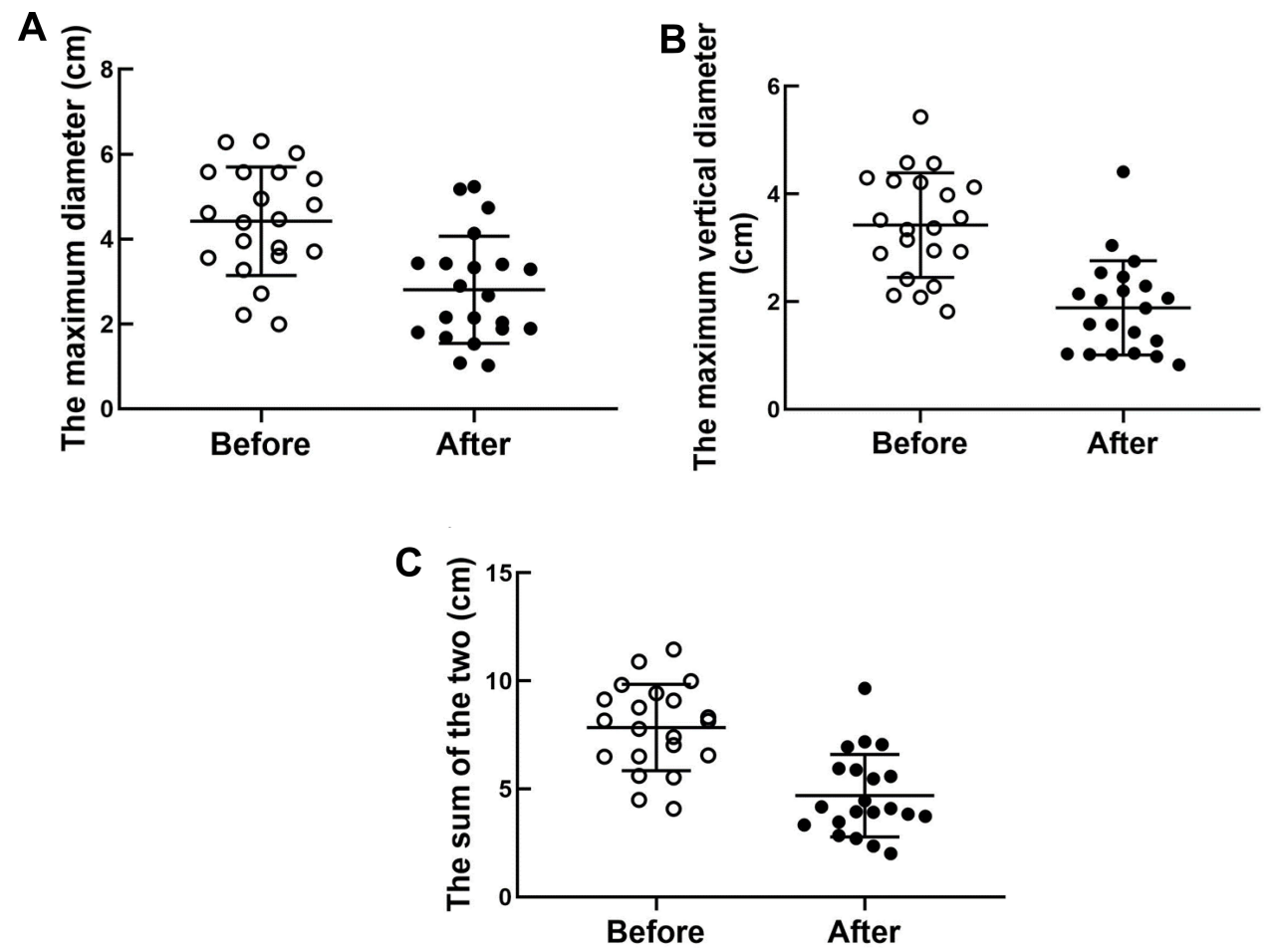

Figure 3 Efficacy of combined-therapy aiming at elderly patients with locally advanced NSCLC diagnosed as tumor invading the chest wall. (A) Compared with baseline, after six months combined-therapy, the maximum diameter of tumor showed a distinct decrease $(4.42 \pm 1.28 \mathrm{vs} 2.81 \pm 1.26 \mathrm{~cm})(P<0.00 \mathrm{I})$. (B) Compared with baseline, after six months combined-therapy, the maximum vertical diameter of tumor showed a distinct decrease $(3.42 \pm 0.97 \mathrm{vs} 1.89 \pm 0.87 \mathrm{~cm})(P<0.000 \mathrm{I})$. (C) Compared with baseline, after six months combined-therapy, the sum of the two of tumor showed a distinct decrease $(7.84 \pm 1.20 \mathrm{vs} 4.69 \pm 1.90 \mathrm{~cm})(P<0.0001)$. 
Table 4 Analysis of Physical Status Score

\begin{tabular}{|l|l|l|l|l|l|}
\hline Group & \multicolumn{4}{|l|}{ ECOG Score } & P-value \\
\hline & $0-1$ & 2 & 3 & 4 & \multirow{2}{*}{$P<0.05$} \\
\cline { 1 - 5 } Before treatment & 3 & 15 & 3 & 0 & \\
\cline { 1 - 5 } After treatment & 18 & 2 & 1 & 0 & \\
\hline
\end{tabular}

patients suffered from esophageal or tracheal fistulae and no cases of late radiation toxicity were observed. Bloody sputum was reported in two patients which cleared with symptomatic treatment. There were four patients with grade $\geq 1$ radiation myelosuppression, and one patient with grade 2 myelosuppression (Table 5).

\section{Discussion}

Primary bronchial lung cancer has remarkably high morbidity and mortality, which threatens the survival outcomes. The majority of NSCLC patients are diagnosed at an advanced stage, thus, losing the chance to undergo surgical resection. Chemotherapy and EBRT are recommended palliative treatment strategies for patients without EGFR or ALK gene mutations. Patients, especially the elderly, decline chemotherapy because of the associated severe adverse effects. EBRT, as an important local treatment approach is associated with irradiation-related side effects which affect the patient's QOL and survival time. $^{23,24}$

$\mathrm{Li}$ et al reported that it was impossible to deliver enough radiation dose in EBRT to eradicate the tumor without damaging the surrounding normal tissues, and especially for tumors measuring $>5 \mathrm{~cm} .{ }^{25}$ However, in clinical practice, physicians recommend the 60Gy prescription dose for primary bronchial lung cancer in EBRT. Besides, the effective radiotherapy dose for tumors with a diameter $>5 \mathrm{~cm}$ should be more than 100Gy as

Table 5 Adverse Effects

\begin{tabular}{|l|l|l|}
\hline Adverse Effects & Cases & Percentage \\
\hline Radiation pneumonitis (grade I) & 1 & 4.7 \\
Radiation esophagitis (grade I) & 2 & 9.5 \\
Pneumothorax & 2 & 9.5 \\
Bloody sputum & 2 & 9.5 \\
Radiation myelosuppression (grade I) & 3 & 14.3 \\
Radiation myelosuppression (grade 2) & 1 & 4.7 \\
Esophageal or tracheal fistulae & 0 & 0 \\
Late radiation toxicity & 0 & 0 \\
\hline
\end{tabular}

reported by Reveizi. ${ }^{6}$ Radiation pneumonitis is a doselimiting adverse effect that may reduce the patient's QOL and is life-threatening. ${ }^{26}$ The elderly population is characterized by poor cardiorespiratory function and physical status. The MILES study pointed out that QOL was a critical factor for the elderly when identifying the appropriate treatment. ${ }^{27}$ Therefore, it is important to identify good treatment strategies for the elderly which take into account the QOL evaluation.

Iodine-125 seed implantation provides an alternative approach to deliver a higher dose (100-170Gy). The radiation dose can fall off sharply within a short distance, and consequently the adjacent normal tissues receive a minimal radiation dose. ${ }^{5}$ Chest wall invasion is defined as soft tissue invasion certified by radiographic evidence and accounts for about $5 \%$ of locally advanced NSCLC. ${ }^{28}$ Considering the relatively high mortality resulting from surgical resection for chest wall invasion within the perioperative period, skilled thoracic surgeons suggest that it is important to identify new strategies. ${ }^{29,30}$ Although stereotactic body radiation therapy (SBRT) can deliver a higher radiation dose to tumor lesions, it is only recommended for small lesions (usually $<5 \mathrm{~cm}$ ). In addition, patients are likely to suffer from rib fracture or chest wall pain several months after SBRT, especially when tumor lesions are adjacent to the chest wall. ${ }^{31,32}$ However, only a few studies report combined-modality therapy; EBRT and iodine-125 seed implantation, in tumor lesions invading the chest wall. In this study, combined-modality therapy showed better local control rate with an ORR and DCR of $71.4 \%$ and $90.5 \%$, respectively at 1 year, in 15 patients (71.4\%) with tumors measuring $>5 \mathrm{~cm}$. Combined-modality therapy is certified not only to deliver a higher dose for chest wall invasion in NSCLC, but to also significantly reduce adverse effects. Iodine-125 seed implantation has limitations. Firstly, it is not easy to perform iodine- 125 seed implantation for lesions located in the mediastinum, and this may lead to an unsatisfactory disposition of iodine-125 seed. Huang and his colleagues reported that it was necessary to carry out artificial pneumothorax for the mediastinum lymph nodes before seed implantation, to meet a good cardiorespiratory function. However, this also increases the risk of complications, such as hemoptysis, chest tightness and dyspnea, which are difficult to tolerate for the elderly. ${ }^{10,32}$ In addition, for large tumors (especially $>5 \mathrm{~cm}$ ), iodine- 125 seed implantation expenses would increase. Therefore, combinedmodality therapy not only reduces the expenses for tumors 
measuring $>5 \mathrm{~cm}$, but also provides a new treatment strategy for tumors invading the chest wall. About $60-90 \%$ of patients with advanced cancer suffer from cancer-related pain, and this is a very common clinical issue. ${ }^{20,33}$ At the same time, the cancer-related symptoms also greatly affect the patients, including dry cough, hoarseness and hemoptysis. Therefore, how to relieve cancer-related pain and ameliorate cancer-related symptoms to improve QOL is a critical question for doctors and patients. In this study, all patients experienced pain caused by the tumor invading the chest wall. A total of $20(95.2 \%)$, experienced complete pain relief after combined-modality. Patients treated with combined-modality therapy did not experience rib fracture or intercostal nerve injury, thus was superior to SBRT. ${ }^{30,31}$ After treatment, the cancer-related symptoms in NSCLC patients were greatly improved, hence, the ECOG performance scores in 18 patients were significantly improved. Song et al indicated that iodine-125 seed implantation ameliorated cancer-related symptoms but also significantly improved QOL in patients diagnosed with advanced NSCLC. ${ }^{34}$ Combinedmodality therapy significantly relieves cancer-related pain and improves ECOG scores which contributes to improved QOL. In this study, severe complications were not reported, which was consistent with the previousreports. $^{3,10,11,16,32}$ Besides, apart from grade 2 irradiation-related myelosuppression (1 case), there were no grade 3 or higher toxicity effects, consistent with a study by Jiang et al, who reported that there was no grade 3 or higher toxicity effects. ${ }^{35}$ A previous study reported that, the incidence of radiation pneumonitis with grade $\geq 2$ was from $7.0 \%$ to $32.0 \%$ of the patients who received EBRT therapy, while the incidence of radiation pneumonitis with grade $\geq 3$ was from 2.6 to $18.0 \%$ of the patients. ${ }^{36-38}$ Thus, it is safe to combine EBRT and iodine-125 seed implantation for locally advanced NSCLC. Due to the lack of prospective randomized controlled trials, it is difficult for clinical physicians to formulate a standardized prescription dose for iodine-125 seed implantation. Previous studies report different tumor volumes, different treatment strategies, and heterogeneity of tumors, thus, it is impossible to obtain effective information about the prescription dose from these studies, which vary from $80 \mathrm{~Gy}$ to $170 \mathrm{~Gy}$. Ji and his colleague reported that a higher prescribed dose correlated with better local control. Besides, they also performed radioactive iodine-125 seed implantation in solid thoracic malignancies, including primary lung cancer or metastatic lung cancer. The median duration of local control was reported to be 11 months with a prescription dose < 140Gy vs 16.4 months with a dose $\geq 140 \mathrm{~Gy}$, though the difference was not significant. A prescription dose exceeding 140Gy for solid thoracic malignancies was strongly suggested, due to the lower marginal recurrence of primary lung cancer. ${ }^{10}$ In our study, the ORR with the tumor receiving a prescription dose $\geq 140 \mathrm{~Gy}$ was found to be better, suggesting that the local control rate should be proportional to the dose delivered to the target lesion. Therefore, this study and consistent with other studies, recommended that the prescription dose for primary lung cancer should exceed 140Gy, and can be higher if well tolerated. However, these results need to be verified through large clinical randomized controlled trials.

\section{Conclusion}

The combined-modality therapy of EBRT and iodine- 125 seed implantation, is a safe and effective treatment option, with minimal adverse effects. Thus, it is recommended as favorable treatment for the elderly with chest wall invasion in locally advanced NSCLC. Combined-modality therapy relieves cancer-related pain, improves the physical status of patients, and contributes to improved QOL. A prescription dose exceeding140Gy may result in better local outcomes. Owing to our limited sample, our conclusion needs to be further clarified.

\section{Acknowledgments}

We thank all participants and patients who participated in this study.

\section{Funding}

This work was supported by the research fund of Binzhou Medical University Hospital foundation (BY2018KJ10). The funding body plays no role in the design, data collection, analysis, interpretation of data in this manuscript.

\section{Disclosure}

The authors report no conflicts of interest in this work.

\section{References}

1. Bray F, Ferlay J, Soerjomataram I, Siegel RL, Torre LA, Jemal A. Global cancer statistics 2018: GLOBOCAN estimates of incidence and mortality worldwide for 36 cancers in 185 countries. CA Cancer J Clin. 2018;68(6):394-424. doi:10.3322/caac.21492

2. Siegel RL, Miller KD, Jemal A. Cancer Statistics, 2017. CA Cancer J Clin. 2017;67(1):7-30. 
3. Wu C, Li B, Sun G, Peng C, Xiang D. Efficacy and safety of iodine- 125 brachytherapy combined with chemotherapy in the treatment of advanced NSCLC in the elderly. Onco Targets Ther. 2018;11:6617-6624. doi:10.2147/OTT.S174457

4. Toschi L, Rossi S, Finocchiaro G, Santoro A. Non-small cell lung cancer treatment (r)evolution: ten years of advances and more to come. Ecancermedicalscience. 2017;11:787. doi:10.3332/ ecancer.2017.787

5. Rong B, Zhao C, Gao W, Yang S. Matrine promotes the efficacy and safety of platinum-based doublet chemotherapy for advanced non-small cell lung cancer. Int $J$ Clin Exp Med. 2015;8 (9):14701-14717.

6. Reveiz L, Rueda JR, Cardona AF. Palliative endobronchial brachytherapy for non-small cell lung cancer. Cochrane Database Syst Rev. 2012;12:CD004284.

7. Ozcan MF, Altinova S, Atan A. Treatment approaches to small renal masses in patients of advanced age ( $>/=75$ years). Turk $J$ Urol. 2018;44(4):281-286. doi:10.5152/tud.2018.04829

8. Xiang GL, Zhu XH, Lin CZ, et al. 125I seed irradiation induces apoptosis and inhibits angiogenesis by decreasing HIF-1alpha and VEGF expression in lung carcinoma xenografts. Oncol Rep. 2017;37 (5):3075-3083. doi:10.3892/or.2017.5521

9. Cheng J, Ma S, Yang G, Wang L, Hou W. The Mechanism of Computed Tomography-Guided 125I Particle in Treating Lung Cancer. Med Sci Monit. 2017;23:292-299. doi:10.12659/MSM.898526

10. Ji Z, Jiang Y, Guo F, et al. Safety and efficacy of CT-guided radioactive iodine- 125 seed implantation assisted by a $3 \mathrm{D}$ printing template for the treatment of thoracic malignancies. $J$ Cancer Res Clin Oncol. 2020;146(1):229-236. doi:10.1007/s00432-019-03050-7

11. Di Maio M, Perrone F. Quality of Life in elderly patients with cancer. Health Qual Life Outcomes. 2003;1:44. doi:10.1186/1477-7525-1-44

12. Dai F, Wang J, An H, et al. Therapy of (125)I particles implantation inhibited the local growth of advanced non-small cell lung cancer: a retrospective clinical study. Am $J$ Transl Res. 2019;11 (6):3737-3749

13. Skowronek J. Brachytherapy in the treatment of lung cancer a valuable solution. J Contemp Brachytherapy. 2015;7(4):297-311. doi: $10.5114 /$ jcb. 2015.54038

14. Qiu H, Ji J, Shao Z, et al. The Efficacy and Safety of Iodine-125 Brachytherapy Combined with Chemotherapy in Treatment of Advanced Lung Cancer: a Meta-Analysis. J Coll Physicians Surg Pak. 2017;27(4):237-245.

15. Huo $\mathrm{X}$, Huo B, Wang $\mathrm{H}$, et al. Implantation of computed tomography-guided Iodine-125 seeds in combination with chemotherapy for the treatment of stage III non-small cell lung cancer. $J$ Contemp Brachytherapy. 2017;9(6):527-534. doi:10.5114/ jcb.2017.72605

16. Chen Y, Li Y, Jia Y, et al. Bronchial artery chemoembolization combined with radioactive iodine-125 seed implantation in the treatment of advanced nonsmall cell lung cancer. $J$ Cancer Res Ther. 2017;13(4):636-641. doi:10.4103/jcrt.JCRT_93_17

17. Li W, Dan G, Jiang J, Zheng Y, Zheng X, Deng D. Repeated iodine-125 seed implantations combined with external beam radiotherapy for the treatment of locally recurrent or metastatic stage III/ IV non-small cell lung cancer: a retrospective study. Radiat Oncol. 2016;11(1):119. doi:10.1186/s13014-016-0688-5

18. Edge SB, Compton CC. The American Joint Committee on Cancer: the 7th edition of the AJCC cancer staging manual and the future of TNM. Ann Surg Oncol. 2010;17(6):1471-1474. doi:10.1245/s10434010-0985-4

19. de Kock I, Mirhosseini M, Lau F, et al. Conversion of Karnofsky Performance Status (KPS) and Eastern Cooperative Oncology Group Performance Status (ECOG) to Palliative Performance Scale (PPS), and the interchangeability of PPS and KPS in prognostic tools. $J \quad$ Palliat Care. 2013;29(3):163-169. doi:10.1177/ 082585971302900305
20. Stewart A, Parashar B, Patel M, et al. American Brachytherapy Society consensus guidelines for thoracic brachytherapy for lung cancer. Brachytherapy. 2016;15(1):1-11. doi:10.1016/j. brachy.2015.09.006

21. Watanabe H, Okada M, Kaji Y, et al. [New response evaluation criteria in solid tumours-revised RECIST guideline (version 1.1)]. Gan to Kagaku Ryoho. 2009;36(13):2495-2501. [Japanese].

22. Licqurish SM, Cook OY, Pattuwage LP, et al. Tools to facilitate communication during physician-patient consultations in cancer care: an overview of systematic reviews. CA Cancer $J$ Clin. 2019;69(6):497-520. doi:10.3322/caac.21573

23. Matsuoka H, Nishio W, Okada M, Sakamoto T, Yoshimura M, Tsubota N. Resection of chest wall invasion in patients with non-small cell lung cancer. Eur J Cardiothorac Surg. 2004;26 (6):1200-1204. doi:10.1016/j.ejcts.2004.07.038

24. Curran WJ, Paulus R, Langer CJ, et al. Sequential vs. concurrent chemoradiation for stage III non-small cell lung cancer: randomized Phase III trial RTOG 9410. J Natl Cancer Inst. 2011;103 (19):1452-1460. doi:10.1093/jnci/djr325

25. Li W, Guan J, Yang L, Zheng X, Yu Y, Jiang J. Iodine-125 brachytherapy improved overall survival of patients with inoperable stage III/IV non-small cell lung cancer versus the conventional radiotherapy. Med Oncol. 2015;32(1):395. doi:10.1007/s12032-0140395-8

26. $\mathrm{Yu} \mathrm{H}, \mathrm{Wu} \mathrm{H}$, Wang $\mathrm{W}$, et al. Machine learning to build and validate a model for radiation pneumonitis prediction in patients with non-small cell lung cancer. Clin Cancer Res. 2019;25 (14):4343-4350. doi:10.1158/1078-0432.CCR-18-1084

27. Gridelli C, Perrone F, Gallo C, et al. Chemotherapy for elderly patients with advanced non-small-cell lung cancer: the multicenter Italian lung cancer in the Elderly Study (MILES) phase III randomized trial. J Natl Cancer Inst. 2003;95(5):362-372. doi:10.1093/ jnci/95.5.362

28. Lanuti M. Surgical management of lung cancer involving the chest wall. Thorac Surg Clin. 2017;27(2):195-199. doi:10.1016/j. thorsurg.2017.01.013

29. Powell JW, Dexter E, Scalzetti EM, Bogart JA. Treatment advances for medically inoperable non-small-cell lung cancer: emphasis on prospective trials. Lancet Oncol. 2009;10(9):885-894. doi:10.1016/ S1470-2045(09)70103-2

30. Doddoli C, D'Journo B, Le Pimpec-barthes F, et al. Lung cancer invading the chest wall: a plea for en-bloc resection but the need for new treatment strategies. Ann Thorac Surg. 2005;80(6):2032-2040. doi:10.1016/j.athoracsur.2005.03.088

31. Nagata Y, Wulf J, Lax I, et al. Stereotactic radiotherapy of primary lung cancer and other targets: results of consultant meeting of the International Atomic Energy Agency. Int J Radiat Oncol Biol Phys. 2011;79(3):660-669. doi:10.1016/j.ijrobp.2010.10.004

32. Huang Q, Chen J, Chen Q, et al. Computed tomographic-guided iodine-125 interstitial implants for malignant thoracic tumors. Eur J Radiol. 2013;82(11):2061-2066. doi:10.1016/j. ejrad.2013.05.037

33. Pan H, Shen P, Shu Q, et al. Efficacy and safety of sustained-release oxycodone compared with immediate-release morphine for pain titration in cancer patients: a multicenter, open-label, randomized controlled trial (SOCIAL). Medicine (Baltimore). 2019;98(24):e15505. doi:10.1097/MD.0000000000015505

34. Song J, Fan X, Zhao Z, et al. (125)I brachytherapy of locally advanced non-small-cell lung cancer after one cycle of first-line chemotherapy: a comparison with best supportive care. Onco Targets Ther. 2017;10:1345-1352. doi:10.2147/OTT.S129903

35. Jiang Y, Ji Z, Guo F, et al. Side effects of CT-guided implantation of (125)I seeds for recurrent malignant tumors of the head and neck assisted by 3D printing non co-planar template. Radiat Oncol. 2018;13(1):18. doi:10.1186/s13014-018-0959-4 
36. Barriger RB, Fakiris AJ, Hanna N, Yu M, Mantravadi P, McGarry RC. Dose-volume analysis of radiation pneumonitis in non-small-cell lung cancer patients treated with concurrent cisplatinum and etoposide with or without consolidation docetaxel. Int J Radiat Oncol Biol Phys. 2010;78(5):1381-1386. doi:10.1016/j. ijrobp.2009.09.030

37. Phernambucq EC, Spoelstra FO, Verbakel WF, et al. Outcomes of concurrent chemoradiotherapy in patients with stage III non-smallcell lung cancer and significant comorbidity. Ann Oncol. 2011;22 (1):132-138. doi:10.1093/annonc/mdq316
38. Werner-Wasik M, Paulus R, Curran WJ, Byhardt R. Acute esophagitis and late lung toxicity in concurrent chemoradiotherapy trials in patients with locally advanced non-small-cell lung cancer: analysis of the radiation therapy oncology group (RTOG) database. Clin Lung Cancer. 2011;12(4):245-251. doi:10.1016/j.cllc.2011.03.026

\section{Publish your work in this journal}

Cancer Management and Research is an international, peer-reviewed open access journal focusing on cancer research and the optimal use of preventative and integrated treatment interventions to achieve improved outcomes, enhanced survival and quality of life for the cancer patient.
The manuscript management system is completely online and includes a very quick and fair peer-review system, which is all easy to use. Visit http://www.dovepress.com/testimonials.php to read real quotes from published authors. 